Check for updates

The BMJ

Cite this as: $B M J 2020 ; 371: \mathrm{m3981}$ http://dx.doi.org/10.1136/bmj.m3981 Published: 15 October 2020

\title{
Long covid could be four different syndromes, review suggests
}

\section{Elisabeth Mahase}

Long covid, the name commonly used to explain lasting effects of covid-19, may actually be four different syndromes, according to a review by the National Institute for Health Research (NIHR). ${ }^{1}$

A team of researchers and doctors reviewed current evidence and interviewed post-hospitalised and non-hospitalised patients and reported that long covid did not seem to fit as one syndrome. They suggested that people experiencing long term effects of covid-19 may have different syndromes such as post-intensive care syndrome, post-viral fatigue syndrome, and long term covid syndrome.

Elaine Maxwell, review author and content lead for NIHR's Centre for Engagement and Dissemination, said, "We are not saying that we have identified four definitive syndromes. We are raising this as a possibility and a possible explanation for why so many people feel they are not being believed or heard and are not getting access to supportive treatments."

NHS England has estimated that up to 5 June, more than 95000 patients had been admitted to hospitals across England with covid-19 and it assumed 45\% would need ongoing support. Figures from the UK Covid Symptom Study app-which has more than four million regular users-suggest that a significant number of people report symptoms for a month and between $10 \%$ and $20 \%$ report complications for longer.

The review highlighted that while data are lacking, several small surveys have reported "remarkably similar findings" that challenge the assumption that most people recover from "mild" infections within two weeks and more serious disease within three weeks.

The surveys showed there are a wide range of recurring symptoms experienced by patients, regardless of whether they were hospitalised, affecting the respiratory system, the brain, cardiovascular system and heart, the kidneys, the gut, the liver, and the skin. The report said these symptoms range in intensity and duration, and do not necessarily present in a linear or sequential manner.

The authors said that the absence of a definition for what patients are experiencing may impact their ability to have their "symptoms and experiences properly recognised and treated by healthcare services, which can, in turn, have a further psychological impact, especially for non-hospitalised patients who were never formally diagnosed."

"It is unclear if all have the same phenomenon. Many researchers and healthcare professionals are cautious about attributing all the reported problems to a single diagnosis," the report said. "However, the lack of a single diagnostic category in no way diminishes the real and often severe continuing impact living with covid-19 has on people's lives. Treatment and support are needed now and are necessarily based on expert consensus and lived experience as well as emergent research findings."

As part of the review, the team also held a focus group with 14 members of the long covid Facebook group. This found that because of diagnostic uncertainty, patients felt that the emphasis on acute respiratory problems "created a misleading impression that anything else was a 'mild case' that would take two weeks recovery."

This binary view combined with the responses people received from healthcare services led to the feeling that the impact of other symptoms was being ignored. In some cases this led to difficulties in patients having their symptoms recognised and treated.

The group said they would prefer a "well informed 'we don't know'" than conflicting messages from different health professionals.

NIHR. Living with covid-19. A dynamic review of the evidence around ongoing covid-19 symptoms (often called long covid). October 2020. https://evidence.nihr.ac.uk/themedreview/living-with-covid19. 